\section{Depresión adulta y experiencias infantiles adversas: evidencia de un subtipo depresivo complejo en consultantes de la atención primaria en Chile}

\author{
VERÓNICA VITRIOL ${ }^{1,2}$, ALFREDO CANCINO $^{1,3}$, \\ MARCELO LEIVA-BIANCHI ${ }^{7, a}$, CARLOS SERRANO ${ }^{7, a}$, \\ SOLEDAD BALLESTEROS ${ }^{4, a}$, SOLEDAD POTTHOFF $^{5}$, \\ CRISTIÁN CÁCERES ${ }^{7, a}$, MARCELA ORMAZÁBAL $^{6, a}$, ANDREA ASENJO $^{3, a}$
}

\section{Association between adverse childhood experiences with depression in adults consulting in primary care}

Background: Traumatic experiences during childhood may influence the development of mental disorders during adulthood. Aim: To determine clinical and psychosocial variables that are associated with a higher frequency of adverse childhood experiences (ACE) in patients who consult for depression in Primary Health Care clinics in Chile. Material and Methods: A socio-demographic interview, the mini international neuropsychiatric interview (MINI), a screening for ACE, a questionnaire for partner violence (PV), the Life Experiences Survey (LES) and the Hamilton Rating Scale for Depression (HRDS) were applied to 394 patients with major depression (87\% women). Results: Eighty two percent of patients had experienced at least one ACE and 43\% of them reported three or more. Positive correlations were observed between the number of ACE and severity of depressive symptoms $(r=0.19 ; p<0.01)$, psychiatric comorbidities $(r=0.23 ; p<0.01)$, partner violence events $(r=0.31 ; p<0.01)$, vital stressful events $(r=0.12 ; p<0.01)$, number of depressive episodes $(r=0.16 ; p<0.01)$, duration of the longer depressive episode $(r=0.12 ; p<0.05)$ and suicidal tendency according to HDRS $(r=0.16 ; p<0.01)$. An inverse correlation was observed between frequency of $A C E$ and age at the first depressive episode $(r=-0.12$; $p<0.05)$. Conclusions: These data are consistent with the hypothesis that early trauma is associated with more severe and complex depressive episodes during adulthood.

(Rev Med Chile 2017; 145: 1145-1153) Stress.

Key words: Child Abuse; Depression; Primary Health Care; Psychological;

\author{
'Escuela de Medicina, Universidad \\ de Talca, Chile. \\ ${ }^{2}$ Hospital San Juan de Dios, \\ Curicó, Chile. \\ ${ }^{3}$ Centro de Salud Familiar Curicó- \\ Centro, Curicó, Chile. \\ ${ }^{4}$ Clínica Privada, Curicó, Chile. \\ ${ }^{5}$ Hospital Regional de Talca, Chile. \\ ${ }^{6}$ Dpto. de Salud Mental, Servicio \\ de Salud del Maule, Talca, Chile. \\ ${ }^{7}$ Escuela de Psicología, \\ Universidad de Talca, Chile. \\ apsicólogo(a) \\ El presente trabajo forma parte \\ del proyecto FONIS SA13/20135 \\ "Factores asociados a las distintas \\ evoluciones que presentan los \\ pacientes que ingresan al GES \\ depresión en los consultorios \\ de la VII Región; seguimiento \\ de una cohorte". FONIS no \\ influyó en el diseño del estudio; \\ en la recolección, análisis o \\ interpretación de los datos; \\ ni en la preparación, revisión \\ o aprobación del presente \\ manuscrito. \\ Los autores declaran conflicto de \\ interés. \\ Recibido el 3 de mayo de 2017, \\ aceptado el 28 de agosto de \\ 2017. \\ Correspondencia a: \\ Verónica Vitriol G. \\ Escuela de Medicina Universidad \\ de Talca Lircay s/n, Talca. \\ Prat 111 Of. 211, Curicó, \\ VII Región, Chile. \\ Teléfono 56-75-2317471 \\ verovitriol@gmail.com \\ vvitriol@utalca.cl
}

E xiste suficiente evidencia que constata que las experiencias infantiles adversas (EIA) constituyen un factor de vulnerabilidad para el desarrollo de patologías mentales y problemas bio-médicos prevalentes en la adultez, tales como: depresión, trastornos de ansiedad, abuso de sustancias, obesidad, hipertensión, diabetes, problemas cardiovasculares, tabaquismo y promiscuidad sexual ${ }^{1-7}$.

Respecto a las patologías mentales la eviden- 
cia sugiere que, comparativamente con quienes no lo reportan, en los pacientes con historia de EIA el trastorno mental emerge más temprano, asociándose a mayor severidad, cronicidad y peor respuesta a los tratamientos estandarizados 8 .

La complejidad psicopatológica que presentan los adultos con antecedentes de EIA se comprende a partir de los hallazgos biológicos que deja la exposición a estrés severo en un período de gran vulnerabilidad para el desarrollo mental. Nemeroff ha sintetizado la evidencia de los últimos 30 años. Ésta da cuenta de cambios epigenéticos, neuroendocrinos, de la neurotransmisión cerebral, del sistema inflamatorio modulado por citoquinas y de daños específicos en áreas cerebrales implicadas en la regulación emocional en sujetos expuestos a EIA?.

Teicher y Samson ${ }^{8}$ postulan la existencia de ecofenotipos,(constelaciones clínicas y neurobiológicas, diferenciadas dentro de cada patología mental, de carácter epigenético, asociados a EIA), que ameritarían un abordaje diferenciado de sus respectivas patologías manifiestas.

Sin embargo, la adversidad biográfica no es indagada en el contexto de la consulta en los servicios de salud ${ }^{10}$, $\mathrm{y}$, según van der Kolk ${ }^{11}$, las devastadoras consecuencias de los EIA sobre la salud adulta aún no están incorporadas en los sistemas diagnósticos vigentes ni en las guías clínico-terapéuticas.

En Chile la depresión es una enfermedad prevalente y discapacitante, y se incluye en las Garantías Explícitas de Salud (GES) desde el año $2006^{12}$. El Ministerio de Salud ha editado guías clínicas con indicaciones de pesquisa, diagnóstico y tratamiento en los diversos niveles de atención ${ }^{13}$. El 90\% de las depresiones son tratadas en la atención primaria de salud (APS), siendo referidas a la especialidad sólo las depresiones psicóticas, refractarias o con intento de suicidio actual $^{13}$.

Por otro lado, un tercio de los adultos chilenos refiere algún $\mathrm{EIA}^{14} \mathrm{y}$, según el último reporte de la UNICEF 71\% de los niños chilenos de 14 años señala haber sufrido algún tipo de violencia ejercida por sus padres ${ }^{15}$. En concordancia con estas cifras nacionales 50 a $80 \%$ de los consultantes por depresión recuerdan al menos un EIA ${ }^{16}$. Más aún, un estudio realizado a nivel secundario constató que los pacientes con depresión severa y poli-trauma infantil ingresaban a tratamiento con un perfil sintomático de mayor severidad, co- morbilidad con TEPT, suicidalidad y dificultades interpersonales ${ }^{17}$.

A pesar de la alta frecuencia de EIA, sin diferencias de género, constatada en consultantes por depresión en la APS de la VII Región de Chile ${ }^{18}$, aún no existen estudios que identifiquen factores clínicos o psicosociales asociados a los EIA en estos usuarios. Lo anterior resulta relevante, ya que estos pacientes son tratados por médicos generales y duplas psicosociales quienes requerirían orientaciones más específicas para identificar a un subgrupo de pacientes depresivos que podría beneficiarse de un abordaje diferenciado.

El objetivo de este trabajo es determinar las variables clínicas y psicosociales asociadas a mayor número de EIA en una muestra de consultantes por depresión en la APS de la VII Región de Chile.

\section{Material y Método}

\section{Diseño}

Estudio cuantitativo descriptivo transversal y correlacional.

\section{Muestra}

De un universo de 2.978 pacientes mayores de 15 años que ingresaron a tratamiento por depresión vía GES en 8 consultorios de APS urbanos de las ciudades de Talca y Curicó, entre febrero y septiembre de 2014, 440 de ellos accedieron a participar en el estudio.

Criterios de exclusión: daño orgánico cerebral, discapacidad sensorial y derivación a atención secundaria por intento de suicidio severo actual, psicosis y/o sospecha de bipolaridad.

Previa firma de un consentimiento informado en mayores de 18 años, o de un asentimiento informado junto al consentimiento del adulto responsable en consultantes entre 15 y 18 años (documentos visados por los Comités de ética e Investigación de la Universidad de Talca (resolución 2013-080) y del Servicio de Salud del Maule (con fecha 13 de diciembre de 2013), los 440 pacientes fueron entrevistados por psiquiatras y psicólogos que procedieron a confirmar el diagnóstico de episodio depresivo de acuerdo a los criterios de la décima versión de la CIE-10, resultando una muestra conformada por 394 pacientes, a quienes el mismo equipo investigador administró una serie de instrumentos. Los pacientes menores de 18 
años $(\mathrm{n}=13)$ fueron entrevistados en forma privada, con la autorización del adulto responsable.

\section{Instrumentos}

- Entrevista clínica semi-estructurada, diseñada por el equipo investigador, que recopila antecedentes sociodemográficos y clínicos, incluyendo patología biomédica crónica.

- Mini Entrevista Neuro-Psiquiátrica Internacional $(M I N I)^{19}$, que indaga los principales trastornos psiquiátricos catalogados en la CIE-10 y la cuarta edición del Manual Diagnóstico y Estadístico de los trastornos mentales (DSMIV).

- Screening de eventos infantiles adversos (EIA), basado en una escala diseñada por Marshall y cols. ${ }^{20}$, ya utilizada en otros estudios chile$\operatorname{nos}^{21,22}$.

- Encuesta de eventos vitales (EV): conocida internacionalmente por la sigla LES (Life Experiences Survey) ${ }^{23}$, traducida al español ${ }^{24}$. Considera 47 ítems que indagan cambios vitales ocurridos durante los últimos 6 meses, tanto positivos como negativos. En esta investigación, se consideró sólo los eventos de connotación negativa.

- Cuestionario de violencia de pareja (VP): 12 preguntas basadas en la definición de la OMS para violencia física, psicológica y sexual ${ }^{25}$.

- Escala para la depresión de Hamilton (HDRS) para cuantificar la severidad de los síntomas ${ }^{26}$.

\section{Análisis estadístico de datos}

Los datos fueron analizados utilizando la decimocuarta versión del programa Statistical Package for the Social Sciences (SPSS). Se realizó estadísticos descriptivos univariados para las características sociodemográficas y clínicas.

Para determinar la relación entre el mayor número de EIA y otras variables continuas, se realizó correlaciones de Pearson bivariadas, incluyendo primero todas las variables, luego sin la variable comorbilidad psiquiátrica (CP) y finalmente considerando una sola CP.

\section{Resultados}

\section{Características sociodemográficas y clínicas}

En la Tabla 1 se puede observar las características sociodemográficas y clínicas de la muestra. En su mayoría son mujeres $(86,8 \%)$, en edad media de la vida, la mitad con pareja y sin actividad remunerada, que iniciaron su primer episodio depresivo en la tercera década de vida, presentando cuadros crónicos y recurrentes, un tercio de ellos con antecedentes de intento de suicidio.

\section{Comorbilidades bio-médicas y psiquiátricas}

El 55\% de los pacientes son portadores de al menos una patología bio-médica.

El $89,1 \%$ de la muestra presenta alguna CP, siendo las más prevalentes: trastorno de pánico $(29,3 \%)$, ansiedad generalizada (25\%), agorafobia (23\%), fobia social (16\%) y estrés post-traumático $(14 \%)$.

\section{Adversidad biográfica}

a) Eventos infantiles adversos. El 82\% de la muestra refirió al menos un EIA, sin diferencias de género respecto a ninguno de los eventos estudiados (Tabla 2). Sólo un adolescente institucionalizado refirió el antecedente de abuso sexual infantil, situación previamente develada.

b) Eventos vitales estresantes en los últimos seis meses. El 21,1\% de la muestra señaló haber vivenciado un solo evento vital y $34,5 \%$ reportó más de tres (Tabla 1).

c) Violencia de pareja. El 58,1\% de la muestra reportó haber sufrido algún tipo de violencia de pareja alguna vez en la vida (58,1\% psicológica; $38,1 \%$ física y $19,8 \%$ sexual).

\section{Relaciones entre variables (Tabla 3)}

Se estableció correlaciones significativas entre mayor número de EIA y: mayor severidad depresiva $(\mathrm{r}=0,19 ; \mathrm{p}<0,01), \mathrm{CP}(\mathrm{r}=0,23 ; \mathrm{p}<0,01)$, VP $(\mathrm{r}=0,31 ; \mathrm{p}<0,01)$, EV $(\mathrm{r}=0,12 ; \mathrm{p}<0,01)$, número de episodios depresivos $(r=0,16 ; p<0,01)$, tiempo de duración del episodio más prolongado $(\mathrm{r}=0,12 ; \mathrm{p}<0,05)$, y suicidalidad de acuerdo a HDRS $(r=0,16 ; p<0,01)$, e inversamente con menor edad del primer episodio depresivo $(\mathrm{r}=-0,12 ; \mathrm{p}<0,05)$.

$\mathrm{Al}$ eliminar de estos análisis la variable $\mathrm{CP}$, se pierden estas asociaciones (Tabla 3 ). Sin embargo, incluyendo sólo una $\mathrm{CP}$, se mantienen las relaciones de EIA con mayor $\mathrm{CP}(\mathrm{r}=0,2 ; \mathrm{p}<0,01)$; $\mathrm{VP}(\mathrm{r}=0,26 ; \mathrm{p}=0,0) ; \mathrm{EV}(\mathrm{r}=0,16 ; \mathrm{p}<0,01)$ y suicidalidad $(\mathrm{r}=0,2 ; \mathrm{p}<0,001)$.

La Figura 1 muestra un gráfico de dispersión entre mayor número de EIA y mayor número de CP. 
Tabla 1. Características sociodemográficas, clínicas y biográficas en 394 pacientes con depresión mayor, APS VII Región, Chile, 2014

\begin{tabular}{|c|c|c|c|}
\hline \multicolumn{4}{|l|}{ Antecedentes sociodemográficos } \\
\hline \multicolumn{2}{|l|}{ Edad al momento de la consulta actual } & $\begin{array}{c}\text { Promedio } \\
47,5 \text { años }\end{array}$ & $\begin{array}{c}\text { DS } \\
15,1\end{array}$ \\
\hline \multicolumn{2}{|l|}{$\begin{array}{l}\text { Sexo } \\
\text { Mujeres } \\
\text { Hombres }\end{array}$} & $\begin{array}{r}\text { n } \\
344 \\
50\end{array}$ & $\begin{array}{c}\% \\
86,8 \\
18,8\end{array}$ \\
\hline \multicolumn{2}{|l|}{$\begin{array}{l}\text { Escolaridad } \\
\text { Sin escolaridad } \\
\text { Básica incompleta } \\
\text { Básica completa } \\
\text { Media incompleta } \\
\text { Media completa } \\
\text { Superior incompleta } \\
\text { Superior completa }\end{array}$} & \begin{tabular}{r}
\multicolumn{1}{c}{$\mathbf{n}$} \\
5 \\
98 \\
53 \\
54 \\
110 \\
30 \\
44
\end{tabular} & $\begin{array}{r}\% \\
1,5 \\
24,8 \\
13,4 \\
13,7 \\
27,9 \\
7,8 \\
11,1\end{array}$ \\
\hline \multicolumn{2}{|l|}{$\begin{array}{l}\text { Estado civil } \\
\text { Soltero } \\
\text { Conviviente } \\
\text { Casado } \\
\text { Separado/Divorciado } \\
\text { Viudo } \\
\text { Vive solo }\end{array}$} & $\begin{array}{r}\mathbf{n} \\
96 \\
49 \\
130 \\
82 \\
39 \\
48\end{array}$ & $\begin{array}{c}\% \\
24,3 \\
12,4 \\
33,0 \\
20,8 \\
9,8 \\
12\end{array}$ \\
\hline \multicolumn{2}{|l|}{ Con actividad remunerada } & 52 & 13,2 \\
\hline \multicolumn{2}{|l|}{ Antecedentes clínicos } & $\begin{array}{c}\text { Promedio } \\
30,7\end{array}$ & $\begin{array}{c}\text { DS } \\
17,2\end{array}$ \\
\hline \multicolumn{2}{|c|}{ Severidad de síntomas depresivos al ingreso, según HDRS (puntos) } & 20 & $\begin{array}{l}4,6 \\
\%\end{array}$ \\
\hline \multicolumn{2}{|l|}{ Historia de intento suicida previo } & 132 & 35 \\
\hline \multicolumn{2}{|l|}{ Promedio de número de episodios depresivos previos } & $\begin{array}{c}\text { Promedio } \\
3,6\end{array}$ & $\begin{array}{l}\text { DS } \\
4,2\end{array}$ \\
\hline \multicolumn{2}{|l|}{ Tiempo promedio del episodio más prolongado (años) } & 3,58 & 7,22 \\
\hline $\mathrm{n}$ de comorbilidades bio-médicas & $\begin{array}{l}0 \\
1 \\
2 \\
3 \text { o más }\end{array}$ & $\begin{array}{c}\mathbf{n} \\
177 \\
84 \\
74 \\
59\end{array}$ & $\begin{array}{l}\quad \% \\
45 \\
21,3 \\
18,8 \\
15\end{array}$ \\
\hline n de comorbilidades psiquiátricas & $\begin{array}{l}0 \\
1 \\
2 \\
3 \\
\text { Más de } 3\end{array}$ & $\begin{array}{r}43 \\
81 \\
80 \\
77 \\
113\end{array}$ & $\begin{array}{l}10,9 \\
20,6 \\
20,3 \\
19,5 \\
28,6\end{array}$ \\
\hline \multicolumn{2}{|l|}{ Antecedentes de adversidad biográfica } & $\mathbf{n}$ & $\%$ \\
\hline $\mathrm{n}$ de eventos vitales estresantes últimos 6 meses 0 & $\begin{array}{l}0 \\
1 \\
2 \\
3 \\
\text { Más de } 3\end{array}$ & $\begin{array}{r}24 \\
83 \\
87 \\
64 \\
136\end{array}$ & $\begin{array}{r}6,1 \\
21,1 \\
22,1 \\
16,2 \\
34,5\end{array}$ \\
\hline $\mathrm{n}$ de eventos de violencia de pareja & $\begin{array}{l}0 \\
1 \\
2 \\
3 \text { o más }\end{array}$ & $\begin{array}{r}165 \\
25 \\
18 \\
186\end{array}$ & $\begin{array}{r}41,9 \\
6,3 \\
4,6 \\
47,2\end{array}$ \\
\hline $\mathrm{n}$ eventos infantiles adversos & $\begin{array}{l}0 \\
1 \\
2 \\
3 \text { o más }\end{array}$ & $\begin{array}{r}71 \\
83 \\
66 \\
174\end{array}$ & $\begin{array}{l}18 \\
21 \\
16,7 \\
44,3\end{array}$ \\
\hline
\end{tabular}


Experiencias infantiles adversas y depresión - V. Vitriol et al

Tabla 2. Frecuencia de eventos traumáticos infantiles en 394 pacientes con depresión, APS, Región del Maule, Chile, 2014

\begin{tabular}{|c|c|c|c|c|c|c|}
\hline \multirow[t]{2}{*}{ ETI } & \multicolumn{2}{|c|}{ Total } & \multicolumn{2}{|c|}{ Mujeres } & \multicolumn{2}{|c|}{ Hombres } \\
\hline & $\mathbf{n}$ & $\%$ & n & $\%$ & $\mathbf{n}$ & $\%$ \\
\hline Separación traumática cuidador & 183 & 46,7 & 163 & 47,7 & 20 & 40 \\
\hline Abuso de alcohol por miembro de la familia & 188 & 48,0 & 165 & 48,2 & 23 & 46 \\
\hline Ser testigo de violencia doméstica & 194 & 49,5 & 170 & 49,7 & 24 & 48 \\
\hline Castigo físico & 176 & 44,9 & 155 & 45,3 & 21 & 42 \\
\hline Secuela castigo físico & 97 & 24,8 & 88 & 25,7 & 9 & 18 \\
\hline Abuso sexual por familiar & 85 & 21,8 & 78 & 22,8 & 7 & 14 \\
\hline Abuso sexual por no familiar & 74 & 19,1 & 64 & 18,7 & 10 & 20,4 \\
\hline A lo menos un tipo de abuso sexual & 131 & 33,2 & 118 & 34,3 & 13 & 26 \\
\hline Los dos tipos de abuso sexual & 26 & 6,6 & 23 & 6,7 & 3 & 6 \\
\hline
\end{tabular}

Tabla 3. Relación entre mayor número de experiencias infantiles adversas y variables clínicas y psicosociales en 394 pacientes con depresión mayor, APS VII región, Chile, 2014

\begin{tabular}{|c|c|c|c|c|c|c|}
\hline \multirow[b]{2}{*}{ Variables } & \multicolumn{2}{|c|}{$\begin{array}{l}\text { Toda la } \\
\text { muestra }\end{array}$} & \multicolumn{2}{|c|}{$\begin{array}{c}\text { Sin } \\
\text { comorbilidad }\end{array}$} & \multicolumn{2}{|c|}{$\begin{array}{c}\text { Con al menos } \\
\text { comorbilidad }\end{array}$} \\
\hline & $\mathbf{R}$ & $\mathbf{p}$ & $\mathbf{R}$ & $\mathbf{p}$ & & $\mathbf{P}$ \\
\hline Edad de consulta actual & $-0,03$ & NS & 027 & NS &,- 019 & NS \\
\hline Edad en primer episodio depresivo & $-0,12$ & 0,05 &,- 066 & NS &,- 105 & NS \\
\hline $\mathrm{n}$ de episodios depresivos & 0,16 & 0,01 & 203 & NS &,- 090 & NS \\
\hline Duración del episodio depresivo más prolongado & 0,10 & 0,05 & 197 & NS &, 064 & NS \\
\hline n de comorbilidades bio-médicas & $-0,03$ & NS &,- 289 & NS & $.0,20$ & NS \\
\hline n de comorbilidades psiquiátricas & 0,23 & 0,01 & - & - & ,206 & 001 \\
\hline n de eventos de violencia de pareja & 0,31 & 0,01 & 055 & NS & ,259 &, 000 \\
\hline n de eventos vitales estresantes durante los últimos seis meses & 0,12 & 0,01 & ,006 & NS &, 161 & 010 \\
\hline Severidad de los síntomas depresivos & 0,19 & 0,01 & 279 & NS & ,090 & NS \\
\hline Suicidalidad & 0,16 & 0,01 & 157 & NS & 204 & ,001 \\
\hline
\end{tabular}

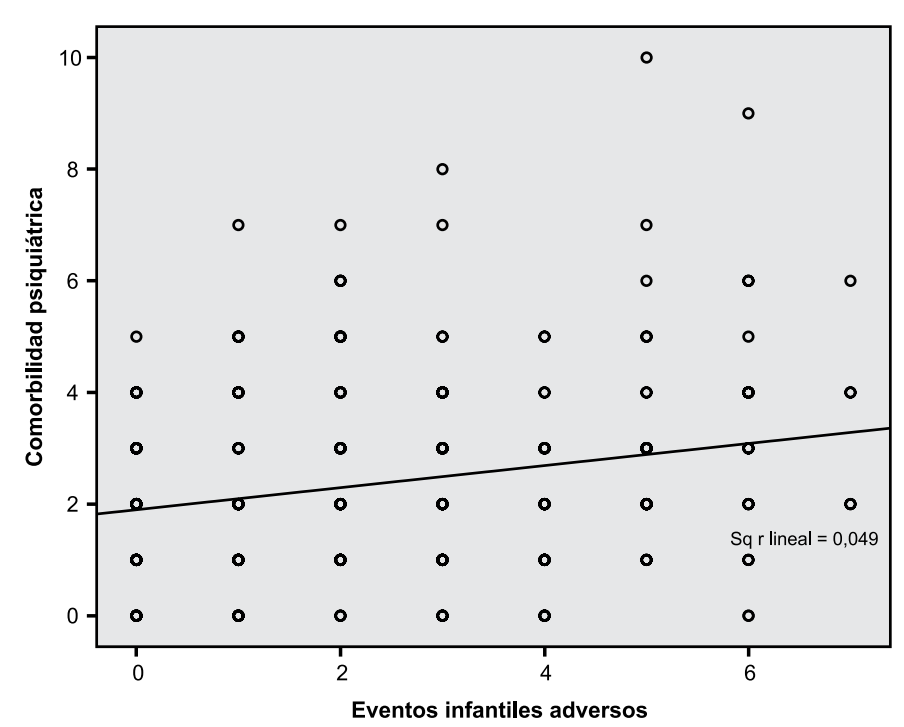

Figura 1. Gráfico de dispersión variable comorbilidad psiquiátrica de acuerdo al MINI y eventos infantiles adversos de acuerdo a escala de Marshall. 


\section{Discusión}

Cerca de la mitad de la muestra señaló haber estado expuesta a tres o más EIA. Lo anterior, guarda concordancia con estudios previos realizados en la VII región ${ }^{16,18}$, pero sobrepasa a $16,5 \%$ reportado por Weil et $\mathrm{al}^{21}$ utilizando el mismo instrumento en una muestra de pacientes con patología bio-médica, y a $9 \%$ reportado por Wingo et $\mathrm{al}^{27}$ en pacientes depresivos estadounidenses. Por otro lado, 33,2\% de exposición a algún tipo de abuso sexual, sin diferencias de género, resulta mayor a las cifras entre $10 \%$ y $20 \%$ reportadas en la literatura, que señala además diferencias significativas de género a favor del sexo femenino ${ }^{28,29}$.

La prevalencia de CP coincide con la literatura respecto a la alta frecuencia de otras patologías mentales que se presentan simultáneamente en pacientes depresivos ${ }^{30-33}$. Sin embargo, $89,1 \%$ de $\mathrm{CP}$ evidenciado en esta muestra resulta superior a lo reportado en otros estudios ${ }^{30-33}$, destacando además la alta proporción de patologías ansiosas. Este resultado se puede atribuir a la alta prevalencia de EIA detectada, considerando que existe evidencia que confirma la relación entre comorbilidades ansiosas y EIA ${ }^{1-3,28}$, e incluso relaciones específicas entre determinadas comorbilidades ansiosas y determinados EIA ${ }^{2,34}$.

El primer análisis entre mayor frecuencia de EIA y las otras variables estudiadas, ratifica en Chile la evidencia respecto a que la depresión con historia de EIA se asocia a un inicio más temprano, mayor severidad al momento de consultar, mayor número de $\mathrm{CP}$, mayor cronicidad y suicidalidad $^{8,28,35-38}$. A esto agregamos la relación de mayor frecuencia de EIA con EV y VP. La relación entre EIA y EV ha sido estudiada en depresión ${ }^{39}$. Sin embargo, de acuerdo a nuestro conocimiento, este sería el primer estudio que constata una relación entre EIA y VP en pacientes depresivos. Este resultado adquiere relevancia ya que en esta muestra encontramos, al igual que Alvarado et $\mathrm{al}^{40}$, una alta frecuencia de VP en consultantes por depresión en Chile.

La mencionada relación entre EIA y los factores mencionados resulta válida al utilizar un análisis bivariado simple. Sin embargo, al eliminar la variable $\mathrm{CP}$, estas relaciones significativas desaparecen. Luego, al incorporar una sola $\mathrm{CP}$, se establecen relaciones significativas sólo entre EIA y mayor CP, VP, EV y suicidalidad. Este resultado concuerda con otros estudios ${ }^{41-43}$, en los cuales, al analizar la relación entre factores clínicos (como comorbilidad o personalidad) y EIA respecto a severidad depresiva, la significación de los EIA establecida en los análisis simples desaparece o disminuye en los análisis multivariados respecto a estas otras variables clínicas ${ }^{41-43}$. Dichos hallazgos están en sintonía con la hipótesis que plantea que los EIA constituyen un factor de vulnerabilidad distal para el desarrollo de la psicopatología adulta y sus complejas consecuencias ${ }^{44}$.

Asimismo, estos resultados orientan al abordaje inicial que podría beneficiar a este subgrupo de pacientes depresivos. De acuerdo a la literatura, la complejidad clínica que presentan los pacientes con psicopatología adulta y antecedentes traumáticos ameritaría un tratamiento diferenciado ${ }^{8}$, por ejemplo, el que propone el modelo de 'tratamiento informado en trauma' (TIT) ${ }^{45}$.

El TIT es un paradigma emergente que reconoce la necesidad de brindar una atención diferenciada en sujetos víctimas de eventos traumáticos cuando consultan a los servicios de salud por problemas mentales o biomédicos crónicos ${ }^{45}$. Entre los postulados del TIT se incluye entregar una capacitación a los médicos generales en el reconocimiento y abordaje de las secuelas que presentan los adultos con historia de trauma, más que una intervención en la historia traumática misma $^{45}$. De acuerdo a esta perspectiva, y según los resultados de este estudio, una estrategia necesaria sería la capacitación a los médicos generales en el reconocimiento de la $\mathrm{CP}$ de tipo ansioso en consultantes por depresión en la APS chilena, más aun considerando que las comorbilidades ansiosas en pacientes con depresión, al igual que los EIA, son sub-diagnosticadas e inadecuadamente tratadas en el contexto de los servicios de salud ${ }^{46}$, lo que se asocia a mayor cronicidad y peor evolución de los cuadros depresivos ${ }^{46}$.

La importancia del componente ansioso en la depresión ha sido reconocida en la sub-tipificación de la ansiedad del DSM- $5^{47}$. La evidencia señala que los cuadros ansiosos se inician a menor edad y suelen preceder a los cuadros depresivos ${ }^{48}$. En base a esto, planteamos que existiría un subtipo de pacientes depresivos, actualmente ingresando a la GES depresión de la APS, cuya patología depresiva se inició más temprano, probablemente precedida por un trastorno de ansiedad y asociada a la presencia de estrés biográfico temprano, dado lo cual, 
la pesquisa de la patología ansiosa en pacientes con depresión adquiere relevancia y requeriría de tratamientos diferenciados ${ }^{49}$.

Es importante destacar que, pese al número de pacientes con patología biomédica de esta muestra, no logramos establecer una relación entre estas patologías y EIA. Lo anterior es un resultado paradojal respecto a los hallazgos del Adverse Childhood Experiences Study (ACES) ${ }^{5}$. Este estudio, replicado en muestras poblacionales de distintos países ${ }^{6,7}$, ha evidenciado una relación entre mayor número de EIA y diversos problemas biomédicos y factores de riesgo para mala salud en general ${ }^{6,7}$. Una hipótesis para explicar nuestros resultados es que el instrumento utilizado consideró sólo algunos de los eventos adversos indagados por el $\mathrm{ACES}^{5}$, hecho que sin duda amerita mayor investigación.

Entre las fortalezas de este estudio podemos señalar que los instrumentos de medición fueron administrados por profesionales con años de experiencia en salud mental. Esto permitió el acceso a información muy confidencial de los eventos adversos biográficos y una evaluación exhaustiva y estandarizada de las distintas comorbilidades psiquiátricas, además de considerar otros factores biográficos adversos como VP y EV.

Entre las limitaciones de este trabajo está el hecho que el instrumento para indagar los EIA fue utilizado en forma retrospectiva y no exploró otros eventos como la negligencia. Respecto a las $\mathrm{CP}$, se excluyó diagnósticos como los trastornos somatomorfos y el único trastorno de personalidad indagado fue el antisocial. Por último, los datos sólo proceden de la región del Maule y, si bien confirman la evidencia internacional respecto a las asociaciones entre EIA y un perfil de pacientes depresivos, podrían no representar la realidad del resto del país.

En resumen, nuestros resultados, que a nuestro entender son los primeros en población chilena y latina, entregan mayor evidencia respecto a un subgrupo de pacientes depresivos que consulta en APS presentando una clínica más compleja que surge de la asociación entre EIA y $\mathrm{CP}$, caracterizada por inicio precoz de la enfermedad depresiva, mayor severidad, suicidalidad y cronicidad de la depresión. Se sugiere continuar investigando este perfil de pacientes depresivos, e incorporar estrategias dirigidas a su reconocimiento y eventual abordaje diferenciado.

\section{Referencias}

1. Spinhoven P, Elzinga BM, Hovens JG, Roelofs K, Zitman FG, van Oppen P, et al. The specificity of childhood adversities and negative life events across the life span to anxiety and depressive disorders. J Affect Disord 2010; 126: 103-12.

2. Cougle JR, Timpano KR, Sachs-Erikson N, Keough ME, Riccardi CJ. Examining the unique relationships between anxiety disorders and childhood physical and sexual abuse in the National Comorbidity Survey-Replication. Psychiatry Research 2010; 177 (1-2): 150-5. doi:10.1016/j.psychres.2009.03.008.[CrossRef], [PubMed], [Web of Science ${ }^{\circledR}$ ] [Google Scholar].

3. Pérez-Benítez C, Vicente B, Zlotnick C, Kohn R, Johnson J, Saldivia S, et al. Estudio epidemiológico de sucesos traumáticos, trastorno de estrés post-traumático y otros trastornos psiquiátricos en una muestra representativa de Chile. Salud mental 2009; 32 (2): 145-53.

4. Norman R, Byambaa M, De R, Butchart A, Scott J, Vos $\mathrm{T}$. The long-term health consequences of child physical abuse, emotional abuse and neglect: a systematic review and meta-analysis. PLoS Medicine 1012; 9 (11): e10001349.

5. Fellitti V, Anda R, Nordenberg D, Williamson D, Spitz A, Edwards V, et al. Relationship of childhood abuse and house hold dysfunction to many of the leading causes of death in adults: The Adverse Childhood Experiences (ACE) Study. Am J Prev Med 1998; 14 (4): 245-58.

6. Bellis M, Lowey H, Leckenby N, Hughes K, Harrison D. Adverse childhood experiences: retrospective study to determine their impact on adult health behaviours and health outcomes in a UK population. J of Public Health 2013; doi:10.1093/pubmed/fdt038.

7. Cuijpers P, Smit F, Unger F, Stikkelbroek Y, Ten Have M, de Graaf R. The disease burden of childhood adversities in adults: a population-based study. Child Abuse \& Neglect 2011; 35 (11): 937-45. doi:10.1016/j. chiabu.2011.06.005.

8. Teicher M, Samson J. Childhood maltreatment and psychopathology: A case for ecophenotypic variants as clinically and neurobiologically distinct subtypes. Am J Psychiatry 2013; 170(10): 1114-33.

9. Nemeroff C. Paradise lost: The neurobiological and clinical consequences of child abuse and neglect. Neuron 2016; 89 (5): 892-909.

10. Sigurdardottir S, Halldorsdottir S. Repressed and silent suffering: consequences of childhood sexual abuse for women's health and well-being. Scand J Caring Sci 2013; 27 (2): 422-32. 
11. van der Kolk B. Commentary: The devastating effects of ignoring child maltreatment in psychiatry-a commentary on Teicher and Samson 2016. J Child Psychol Psychiatry 2016; 57 (3): 267-70.

12. Salvo L. Magnitud, impacto y estrategias de enfrentamiento de la depresión, con referencia a Chile. Rev Med Chile 2014; 142: 1157-64.

13. Ministerio de Salud. Guía Clínica Depresión en personas de 15 años y más. Santiago, MINSAL, 2013. http:// www.minsal.cl/portal/url/item/7222754637c08646e04001011f014e64.pdf

14. Zlotnick C, Johnson J, Kohn R, Vicente B, Rioseco P, Saldivia S. Epidemiology of trauma, post-traumatic stress disorder (PTSD) and comorbid disorders in Chile. Psychol Med 2006; 36 (11): 1523-33.

15. Larraín S, Bascuñán C. IV Estudio de Maltrato Infantil, UNICEF, 2012.

16. Vitriol V, Cancino A, Weil K, Salgado C, Asenjo M, Potthoff S. Depression and psychological trauma: an overview integrating current research and specific evidence of studies in the treatment of depression in public mental health services in Chile. Depress Res Treat 2014; 1-10. doi.org/10.1155/2014/608671.

17. Ballesteros S, Vitriol V, Florenzano R, Calderón A, Vacarezza A. Mujeres con depresión severa: relación entre trauma infantil y gravedad de síntomas clínicos. Rev Chil Neuro-Psiquiatr 2007; 45 (4): 288-95.

18. Cancino A, Asenjo M, Jofré X. Estudio de antecedentes traumáticos infantiles en personas ingresadas al programa de depresión en el CES Curicó-Centro durante el año 2006. Rev FACE-ESGS 2007; 3, http://www. cegis.utalca.cl/doc/Publicaciones/ESGS/ESGS_a3_N7/ FACE_ESGS_3_7.pdf

19. Sheehan D, Lecrubier Y, Sheehan K, Amorim P, Janavs J, Weiller E, et al. The Mini International Neuropsychiatric Interview (MINI): The development and validation of a structured diagnostic psychiatric interview for DSM-IV and ICD-10. J Clin Psychiatry 1998; 59 (20): 22-33.

20. Marshall R, Schneier F, Lin S, Simpson H, Vermes D, Liebowitz M. Childhood trauma and dissociative symptoms in Panicdisorder. Am J Psychiatry 2000; 157 (3): 451-3.

21. Weil K, Florenzano R, Vitriol V, Cruz C, Carvajal C, Fullerton C, et al. Child battering and adult psychopathology: an empiric study. Rev Med Chile 2004; 132: 1499-504.

22. Vitriol V, Ballesteros S, Florenzano R, Weil K, Benadof D. Evaluation of an out patient intervention for women with severe depression and a history of childhood trauma. Psychiatr Serv 2009; 60: 636-942.
23. Sarason I, Johnson J, Siegel J. Assessing the impact of life changes: Development of the Life Experiences Survey. J Consult Clin Psychol 1978; 46 (5): 932-46.

24. Páez D, et al. Salud Mental y Factores Psicosociales. 1986. Madrid: Fundamentos.

25. Illanes E, Bustos L, Vizcarra B, Muñoz S. Violencia y factores sociales en mujeres de la ciudad de Temuco. Rev Med Chile 2007; 135: 326-34.

26. Ramos-Brieva JC, Cordero-Villafafila A. A new validation of the Hamilton Rating Scale for Depression. J Psychiatr Res 1988; (22): 21-8.

27. Wingo A, Wrenn G, Pelletier T, Gutman A, Bradley B, Ressler K. Moderating effects of resilience in individuals with childhood abuse o trauma exposure. J Affect Disord 2010; 126 (3): 411-4.

28. Hovens J, Wiersma J, Giltay E, Van Oppen P, Spinhoven P, Penninx B, et al. Childhood life events and childhood trauma in adult patients with depressive, anxiety and comorbid disorders vs. controls. Acta Psychiatr Scand 2010; 122 (1): 66-74.

29. Koola M, Qualls C, Kelly D, Skelton K, Bradley B, Amar $\mathrm{R}$, et al. Prevalence of childhood physical and sexual abuse in veterans with psychiatric diagnoses. J Nerv Ment Dis 2013; 201 (4): 348-52. doi:10.1097/NMD. 0b013e318288e333.

30. Howland R, Rush A, Wisniewski S, Trivedi M, Warden $\mathrm{D}$, Fava M, et al. Concurrent anxiety and substance use disorders among out patients with major depression: Clinical features and effect on treatment outcome. Drug Alcohol Depend 2009; 99 (1-3): 248-60.

31. Lamers F, van Oppen P, Comijsm H, Smit J, Spinhoven $\mathrm{P}$, van Balkom A, et al. Comorbidity patterns of anxiety and depressive disorders in a large cohort study: the Netherlands Study of Depression and Anxiety (NESDA). J Clin Psychiatry 2011; 72 (3): 341-8.

32. Thaipisuttikul $P$, Ittasakul $P$, Waleeprakhon $P$, Wisajun $\mathrm{P}$, Jullagate S. Psychiatric comorbidities in patients with major depressive disorder. Neuropsychiatr Dis Treat 2014; 10 (10): 2097-103.

33. Martínez P, Rojas G, Fritsch R, Martínez V, Vohringer $\mathrm{P}$, Castro A. Comorbilidad en personas con depresión que consultan en centros de la atención primaria de salud en Santiago, Chile. Rev Med Chile 2017; 145 (1): 25-32.

34. Vitriol V, Cancino A, Leiva-Bianchi M, Serrano C, Ballesteros S, Asenjo A, et al. Childhood trauma and psychiatric comorbidities in patients with depressive disorder in primary care in Chile. J Trauma Dissociation 2017; 8 (2): 189-205. doi:10.1080/15299732.2016.12124 49.

35. Bernet C, Stein M. Relationship of childhood maltreat- 
ment to the onset and course of major depression in adulthood. Depress Anxiety 1999; 9: 169-74.

36. Hovens J, Giltay E, Wiersma J, Spinhoven P, Penninx B, Zitman F. Impact of childhood life events and trauma on the course of depressive and anxiety disorders. Acta Psychiatr Scand 2012; 126 (3): 198-207. [PubMed: 22268708].

37. Nanni V, Uher R, Danese A. Childhood maltreatment predicts unfavorable course of illness and treatmento utcome in depression: a meta-analysis. Am J Psychiatry 2012; 169 (4): 141-51.

38. López-Castroman J, Melhem N, Birmaher B, Greenhill B, Kolko D, Stanley B, et al. Early childhood sexual abuse increases suicidal intent. World J Psychiatry 2013; 12 (2): 149-54. doi:10.1002/wps.20039.

39. Korkeila J, Vahtera J, Nabi H, Kivimäki M, Korkeila K, Sumanen M, et al. Childhood adversities, adult hood life events and depression. J Affect Disord 2010; 127 (1-3): $130-8$.

40. Alvarado R, Vega J, Sanhueza G, Muñoz G. Evaluación del programa para la detección, diagnóstico y tratamiento Integral de la depresión en atención primaria en Chile. Rev Panam Salud Pública 2005; 18 (4-5): 278-86.

41. Spijker J, de Graaf R, Ten Have M, Nolen W, Speckens A. Predictors of suicidality in depressives pectrum disorder in the general population: results of the Netherlands Mental Health Survey and Incidence Study. Soc Psichiatry Psychiat Epidemiol 2010; 45: 513-21.

42. Hayashi Y, Okamoto Y, Tagakaki K, Okada G, Toki S, Inoue $\mathrm{T}$, et al. Direct and indirect influences of childhood abuse on depression symptoms in patients with major depressive disorder. BMC Psychiatry 2015; 15 (1): e244.

43. Vitriol V, Cancino A, Ballesteros S, Potthoff S, Serrano S. Factors associated with greater severity of depression in Chilean primary care. Prim Care Companion CNS Disord 2017; 19 (3) doi:10.4088/PCC.16m02051.

44. Clark C, Caldwell T, Power C, Stansfeld SA. Does the influence of childhood adversity on psychopathology persist across the life course? A 45-year prospective epidemiologic study. Annals of Epidemiology, 20(5): 385-394. doi:10.1016/j.annepidem.2010.02.008

45. Raja S, Hasnain M, Hoersch H, Gove-Yin S, Rajagopalan $\mathrm{S}$. Trauma informed, care in medicine: current knowledge and future research directions. Fam, Community, Health 2015; 38(3): 216-26.

46. Hirschfeld R. The comorbidity of major depression and anxiety disorders: Recognition and management in primary care. Prim Care Companion J Clin Psychiatry 2001; 3 (6): 244-54.

47. Gaspersz R, Lamers F, Kent J, Beekman A, Smit J, van Hemert A, et al. Longitudinal predictive validity of the DSM-5 anxious distress specifier for clinical outcomes in a large cohort of patients with mejor depressive disorder. J Clin Psychiatry 2017; 78 (2): 207-13.

48. deGraaf R, Bijl R, Smit F, Vollebergh W, Spijker J. Riskfactorsfor 12-month comorbidity of mood, anxiety and substance use disorders: findings from the Netherlands Mental Health Survey and Incidence Study. Am J Psychiatry 2002; 159 (4): 620-9.

49. Coplan J, Aaronson C, Panthanghi V, Kim Y. Treating comorbid anxiety and depression: Psychosocial and pharmacological approaches. World J Psychiatry 2015; 5 (4): 366-78. 